\title{
Primary Extranodal Hodgkin's Lymphoma presenting with a Single Pelvic Mass
}

\author{
${ }^{1}$ Gurkan Kiran, ${ }^{2}$ Emel Canaz, ${ }^{3}$ Hamide Sayar, ${ }^{4}$ Salih Serin, ${ }^{5}$ Ozan Balakan
}

\begin{abstract}
Introduction: Although lymphomas can arise from a wide variety of tissues, primary extranodal presentation is predominantly a feature of non-Hodgkin's lymphomas. Primary pelvic extranodal Hodgkin's lymphoma is a rare malignancy. We presented a case of primer retroperitoneal Hodgkin's lymphoma initially presumed gynecologic malignancy. We discussed its rarity, staging and management.
\end{abstract}

Case report: A 75-year-old woman was referred to our hospital with persisting pelvic mass. Pelvic examination revealed an ill-defined immobile mass with hard in consistency. The mass $100 \times 75 \mathrm{~mm}$ in diameter, invading right parametrium, perivesical space and bladder wall was detected in CT and there were no signs of metastases or enlarged lymph nodes. Diagnostic laparoscopy was suggested but the patient refused the surgery due to her advanced age. One year later, she presented with the symptoms of fever, sweats and fatigue. Hodgkin's lymphoma was diagnosed via laparoscopic biopsy of the mass located at retroperitoneal space anteriorly to the uterus. CT examination revealed multiple swollen lymph nodes and a pulmonary nodule; thus, stage 4 disease was established. International prognostic score was 2 , indicating $67 \%$ progression free survival; however, patient died due to respiratory failure after the first course of ABVD chemotherapy.

Conclusion: Although gynecologists rarely experience primary extranodal lymphomas particularly Hodgkin's disease, lymphoid malignancies should be kept in mind in differential diagnosis of pelvic masses. This disease can rarely arise adjacent to gynecologic organs in retroperitoneal space, and this presentation can be misinterpreted as a gynecological malignancy.

Keywords: Pelvic mass, Extranodal lymphoma, Hodgkin's disease.

How to cite this article: Kiran G, Canaz E, Sayar H, Serin S, Balakan O. Primary Extranodal Hodgkin's Lymphoma

${ }^{1}$ Professor, ${ }^{2}$ Fellow, ${ }^{3,5}$ Associate Professor, ${ }^{4}$ Medical Doctor

1,2,4 Department of Obstetrics and Gynecology, Kahramanmaras Sutcu Imam University School of Medicine, Kahramanmaras Turkey

${ }^{3}$ Department of Pathology, Kahramanmaras Sutcu Imam University School of Medicine, Kahramanmaras, Turkey

${ }^{5}$ Division of Medical Oncology, Department of Internal Medicine, Kahramanmaras Sutcu Imam University School of Medicine, Kahramanmaras, Turkey

Corresponding Author: Emel Canaz, Department of Obstetrics and Gynecology, Kahramanmaras Sutcu Imam University School of Medicine, Kahramanmaras-46000,Turkey Phone: +903442803211, e-mail: emelcanaz@gmail.com presenting with a Single Pelvic Mass. J South Asian Feder Obst Gynae 2014;6(3):183-186.

Source of support: Nil

Conflict of interest: None

\section{INTRODUCTION}

The survival rate of Hodgkin's lymphoma is increasing constantly, with a median cure rate of $90 \%{ }^{1}$ The presence of bulky and advanced stage disease are the leading prognostic factors for treatment failure and recurrence. Occurrence at unexpected locations may cause delay in the diagnosis and progression of the disease. There are increasing numbers of case reports presenting extranodal non-Hodgkin's lymphomas in pelvic area, but it is limited for Hodgkin's lymphoma. Here, we present case of a pelvic mass presumed gynecologic malignancy and diagnosed as primary pelvic extranodal Hodgkin's lymphoma.

\section{CASE REPORT}

A 75-year-old postmenopausal woman was referred to our hospital with the suspicion of a gynecologic malignancy. She had a history of a pelvic mass that had been neglected for 2 years, but no history of explorative surgery. Bimanual pelvic examination revealed right adnexal immobile mass with hard in consistency. Ultrasonographic findings were compatible with a heterogeneous right adnexal hypoechoic lesion. A few weeks ago, she was admitted to another center with the symptoms of pelvic pain and vaginal bleeding. Abdominopelvic computed tomography (CT) was used to display a $100 \times$ $75 \mathrm{~mm}$ lobulated mass adjacent to uterus, with irregular demarcation lines and calcifications inside. The mass invaded the right parametrial and paravesical space and the bladder wall, encompassing the right ureter. There was grade 2 hydroureteronephrosis in right kidney and no signs of abdominal metastases or swelling in lymph nodes. She was then referred to our hospital with the suspicion of gynecologic malignancy. Thorax CT examination was used to scan for distant metastasis and no any pulmonary nodules or enlarged lymph nodes were detected. Laboratory data showed normal levels of creatinine $(0.79 \mathrm{mg} / \mathrm{dl} ; 0.2-1.3 \mathrm{mg} / \mathrm{dl})$ elevated levels of BUN (29 $\mathrm{mg} / \mathrm{dl} ; 6-20 \mathrm{mg} / \mathrm{dl})$, an elevated sedimentation rate 


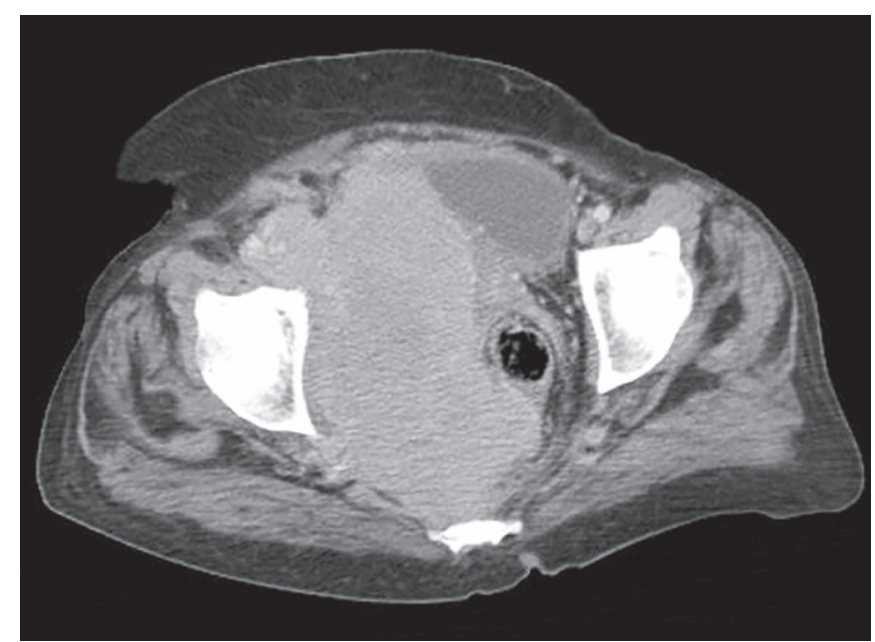

Fig. 1: Axial computerized tomography scan of the pelvic cavity. A lobulated mass with irregular borders extending into right parametrial and paravesical tissues is present. Posterolateral wall irregularities can be seen in the bladder

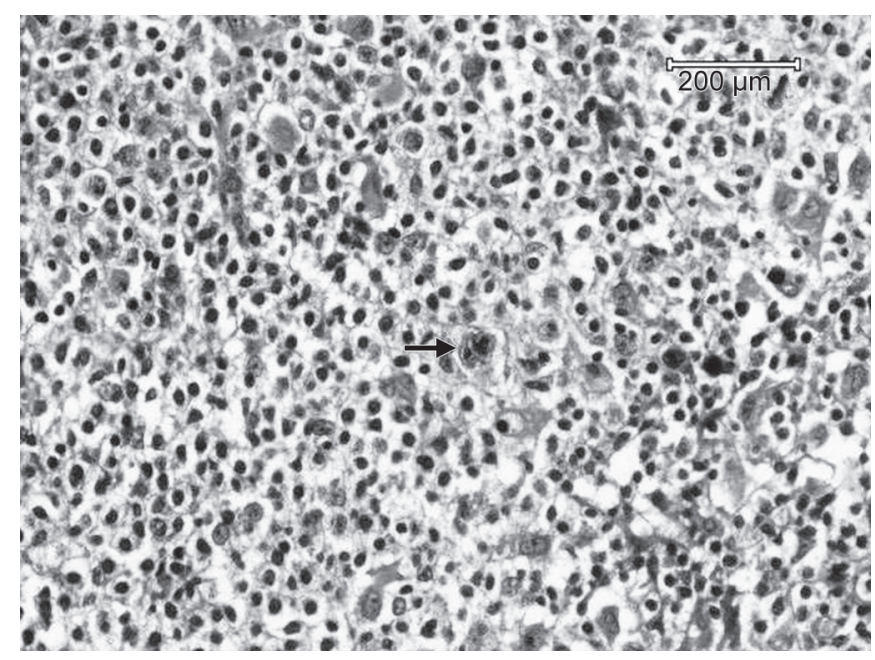

Fig. 3: Microscopic appearance of the tumor. Large malignant cell known as Reed-Sternberg (arrow) admixed within a reactive cell infiltrate is demonstrated

(58 mm/h; 0-30 mm/h), and elevated CRP $(8.63 \mathrm{mg} / \mathrm{l}$; $0-5 \mathrm{mg} / \mathrm{l})$. CA 125 assay revealed a slight elevation (44.7 $\mathrm{U} / \mathrm{ml} ; 5-35 \mathrm{U} / \mathrm{ml})$. Under suspicion of a gynecological malignancy, endometrial and endocervical sampling was performed prior to scheduled laparoscopic surgery. But the patient declined the surgery due to her advanced age. Histological analysis showed that endometrium and endocervix was tumor free.

One year later, the patient was readmitted to our hospital with symptoms of progressive fatigue, fever and night sweats. Evaluation by abdominopelvic and thoracic CT revealed that the lesion had expanded, it was $150 \times 80 \mathrm{~mm}$ in diameter (Fig. 1). Computed tomography also showed a subpleural pulmonary nodule $4 \mathrm{~mm}$ in diameter, and apparent lymphadenopathy in pelvis, para-aortic area, inguinal regions and mediastinum. An ultrasonography-guided percutaneous core-needle biopsy was administered to the pelvic mass, but histo-

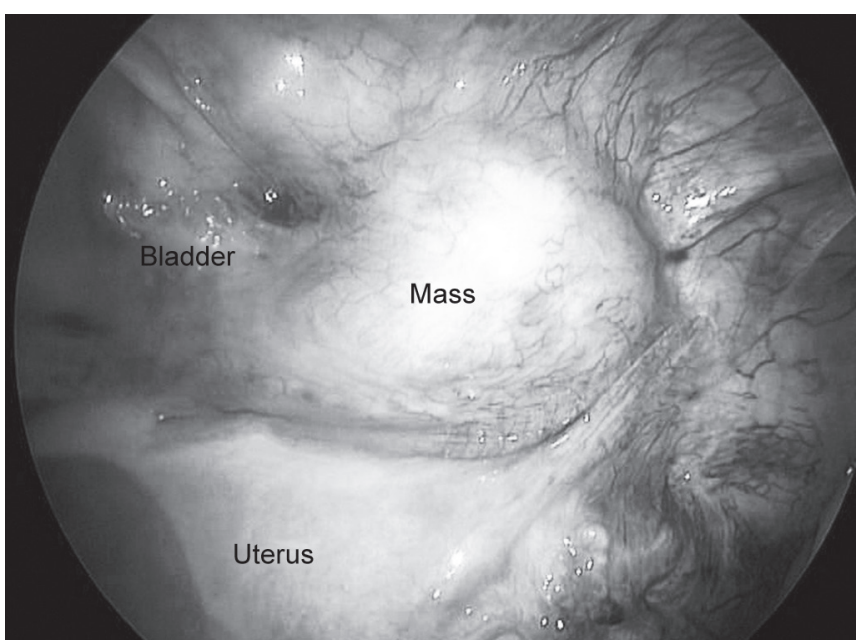

Fig. 2: Laparoscopic view of the mass

pathologic examination was nondiagnostic with 'material including lymphoid cells', not showing apparent malignancy. Laparoscopy was performed and it exposed an atrophic retroverted uterus with small ovaries and a mass at retroperitoneal space anteriorly to the uterus. Boundaries of the mass were right pubic bone anteriorly, right round ligament in the right side and uterus posteriorly; medial boundary was not easily distinguished from the bladder (Fig. 2). Sampling of the tumoral mass was carried out with monopolar scissors and plasma kinetic forceps. An injury occurred on the bladder wall while dissecting the mass and the laceration was primarily repaired with a single noninterrupted absorbable suture. Preliminary histological study at frozen section revealed the suspicion of lymphoma, and no further surgery was performed.

Immunohistochemical studies confirmed the classical Hodgkin's lymphoma, lymphocyte-rich type (Fig. 3). Immunostaining of Reed-Sternberg cells showed focal CD30 positivity and CD15 negativity. Reactive lymphoid cells showed CD3, CD20, and LCA positivity. Complete clinical staging by means of $\mathrm{CT}$ scans of the abdomen and thorax, showed stage 4 disease according to the Ann Arbor classification. PET/CT showed abnormal uptake at all mentioned lesions with a SUV level of 9.9 in the pelvic mass. International prognostic score was 2 with low hemoglobin level $(8.5 \mathrm{gm} / \mathrm{dl})$ and stage 4 disease; other risk factors were not present (serum albumin was $4.4 \mathrm{gm} / \mathrm{dl}$, white cell count was $7890 / \mathrm{mm}^{3}$ and lymphocyte count was $20.7 \%$ of white cell count). Human immunodeficiency virus (HIV) serostatus was assessed with ELISA, and the patient was seronegative (0.03). Six courses of cytotoxic chemotherapy consistent with the ABVD protocol (adriamycin, bleomycin, vincristine, dacarbazine) was planned on a monthly basis. Unfortunately, after the first course of chemotherapy, the patient's condition deteriorated, and she died due to respiratory failure. 


\section{DISCUSSION}

Hodgkin's lymphoma is predominantly a nodal disease, and the most common presentation is indolent lymphadenopathy. Primary extranodal disease with a single pelvic mass without systemic involvement is a rare condition. ${ }^{2}$ If lymphoma arises in a single organ and confined to the adjacent lymphoid tissue, it is termed as primary. In the presence of more than one afflicted organ or extranodal site, or involvement of nodes other than just contiguous with primary organ, it is termed as secondary. Usually, secondary extranodal lymphoma is more common than primary extranodal lymphoma and extranodal disease is more common in non-Hodgkin (20-40\%) than Hodgkin's lymphoma (4-5\%). ${ }^{3}$ The latter may be rarer as $0.25 \% .{ }^{4}$ Initial presentation of the current case was compatible with primary extranodal disease arised in anterior retroperitoneum without metastases and enlarged lymph nodes in CT. And findings at re-admittence demonstrates the dissemination of the disease during one year that the patient lost to follow.

Lymphomas can arise from various organs, and this was attributed to lymphoid tissue in organs and migration in a particular fashion. ${ }^{3}$ Retroperitoneal presentation is predominantly a feature of non-Hodgkin's lymphomas. ${ }^{5}$ Although the most rational diagnosis was a retroperitoneal tumor, we can not precisely rule out the bladder originating neoplasms. In their presentation, Sonmezer et al reported a primary non-Hodgkin lymphoma of urinary bladder presenting as a large pelvic mass located anteriorly to the uterus and extending into right adnexal space as in the current case. ${ }^{6}$ As we could access, there are two case reports in the English literature presenting primary bladder Hodgkin's lymphoma. ${ }^{7,8}$ Neverthless, retroperitoneal tumors also invade the neighboring organs frequently and cystoscopy was missing to make such a diagnosis.

When the diagnosis of lymphoma is made, referral to medical oncology is essential for proper treatment. Staging in both Hodgkin's and non-Hodgkin's disease is established on the basis of the Ann Arbor staging system by imaging, preferably with CT, MRI or PET. Table 1 summarizes the Ann Arbor staging system for extranodal lymphomas. Stage IE and IIE tumors are reliably considered as primary extranodal lymphoma, but in stage IIIE and IV it is difficult to distinguish primary extranodal disease from seconder involvement of disseminated lymphoma, unless the history is known as in the presented case. Although there is no pathognomonic symptoms, fever, weight loss, night sweats also known as 'B symptoms', are significantly present in the advanced disease. ${ }^{10}$ When evaluated retrospectively, the patient's
Table 1: Ann Arbor staging system for extranodal lymphomas

\begin{tabular}{|c|c|}
\hline Stage & Area of involvement \\
\hline IE & One extralymphatic site involvement \\
\hline IIE & $\begin{array}{l}\text { One extralymphatic organ and one or more lymph node } \\
\text { regions involvement on the same side of the diaphragm }\end{array}$ \\
\hline IIIE & $\begin{array}{l}\text { One extralymphatic organ and lymph node regions } \\
\text { involvement on both sides of the diaphragm }\end{array}$ \\
\hline IV & $\begin{array}{l}\text { Diffuse or disseminated involvement of one or more } \\
\text { extralymphatic organs, including any involvement of the } \\
\text { liver, bone marrow or nodular involvement of the lungs }\end{array}$ \\
\hline
\end{tabular}

stage at the time of her initial admission was $1 \mathrm{E}$ according to Ann Arbor staging system and it is stated as 4, one year later. The recommended management of early stage Hodgkin's lymphoma is two courses of ABVD chemotherapy, followed by involved-field radiotherapy with a radiation dose of 20-Gy. ${ }^{11}$ This strategy has achieved a 5 years overall survival rate of $94 \%$. Patients with advanced state disease are managed with cytotoxic therapy according to the BEA-COPP (bleomycin, etoposide, doxorubicin, cyclophosphamide, vincristine, procarbazine, prednisone) protocol; consolidation radiotherapy with 30-Gy radiation dose of PET-positive residual mass $\geq 2.5 \mathrm{~cm}$ is strongly recommended. ${ }^{1}$ This regimen yields a 5 -year overall survival rate of $95.3 \%$. Patients over 60 years old and advanced stage disease may be treated with six to eight courses of ABVD or PVAG (prednisone, vinblastine, adriamycin, gemcitabine) polychemotherapy, followed by 30-Gy radiotherapy of residual mass $>1.5 \mathrm{~cm}$ and never be managed with BEA-COPP regimen because of its high acute toxicity. ${ }^{12}$ International prognostic score on advanced stage disease can predict the disease free survival; a score of 3 or more represents a moderate-high risk with an expected $55 \%$ rate of progression free survival and $70 \%$ overall survival in a 5 -year period. ${ }^{13}$ As in represented case, prognostic score 2 corresponds to an expected $67 \%$ progression free survival and $81 \%$ overall survival. However, prognostic score does not eliminate the toxic effects of chemotherapy which is the probable cause of death in this patient.

It has been reported that malignant lymphoma can be associated with elevated levels of CA 125. This can contribute to misinterpretation of findings in the setting of a newly diagnosed pelvic mass. Higher levels of CA 125 have been attributed to secretion from stimulated serosal mesothelial cells by cytokines released from lymphoid tissue. ${ }^{14}$ CA 125 secretion from malignant lymphoid cells has not been shown. Furthermore, CA125 correlates with clinical stage and disease aggressiveness, and may be useful in disease monitoring. ${ }^{15}$ We noted slight elevation in CA125 levels in our case. Despite the current case was not associated with HIV infection, some reports draw attention to the relationship between extranodal 
lymphomas and HIV infection and suggest evaluation of HIV status in the patients with extranodal lymphomas. Literature findings also support the greater risk in HIV infected patients for extranodal lymphomas rather than the diseases confined to the lymphoreticular system. ${ }^{16-18}$

Although gynecologists rarely experience primary extranodal lymphomas particularly Hodgkin's disease, differential diagnosis of pelvic masses should include lymphoid malignancies. Familiarity and suspicion may prompt an earlier diagnosis which can bring about a better prognosis.

\section{REFERENCES}

1. Engert A, Haverkamp H, Kobe C, et al. Reduced-intensity chemotherapy and PET-guided radiotherapy in patients with advanced stage Hodgkin's lymphoma (HD15 trial): a randomised, open-label, phase 3 non-inferiority trial. Lancet 2012;379(9828):1791-1999.

2. Young GAR. Lymphoma at uncommon sites. Hematol Oncol 1997;17(2):53-83.

3. Charnsangavej C. Lymphoma of the genitourinary tract. Radiol Clin North Am 1990;28(4):865-877.

4. Wood NL, Coltman CA. Localized primary extranodal Hodgkin's disease. Ann Intern Med 1973;78(1):113-118.

5. Chen L, Kuriakose P, Hawley RC, et al. Hematologic malignancies with primary retroperitoneal presentation. Clinicopathological study of 32 cases. Arch Pathol Lab Med 2005; 129(5):655-660.

6. Sonmezer M, Ensari A, Ustun Y, et al. Primary lymphoma of the urinary bladder presenting as a large pelvic mass. J Pak Med Assoc 2002;52(5):228-230.

7. Marconis JT. Primary Hodgkin's (paragranulomatous type) disease of the bladder. J Urol 1959;81(2):275-281.
8. Lebowich J. Hodgkin's disease involving the bladder: Report of a case. Am J Cancer 1937;30(4):758-764.

9. Carbone PP, Kaplan HS, Musshoff K, et al. Report of the Committee on Hodgkin's Disease Staging Classification. Cancer Res 1971;31(11):1860-1861.

10. Bedewy M, El-Maghraby S, Bedewy A. CD163 and c-Met expression in the lymph node and the correlations between elevated levels of serum free light chain and the different clinicopathological parameters of advanced classical Hodgkin's lymphoma. Blood Res 2013;48(2):121-127.

11. Engert A, Plutschow A, Eich HT, et al. Reduced treatment intensity in patients with early-stage Hodgkin's lymphoma. N Engl J Med 2010;363(7):640-652.

12. Engert A, Ballova V, Haverkamp H, et al. Hodgkin's lymphoma in elderly patients: a comprehensive retrospective analysis from the German Hodgkin's Study Group. J Clin Oncol 2005;23(22):5052-5060.

13. Hasenclever D, Diehl V. A prognostic score for advanced Hodgkin's disease. N Eng J Med 1998;339(21):1506-1514.

14. Zeillemaker AM, Verbrugh HA, Hoynck van Papendrecht AA, et al. CA 125 secretion by peritoneal mesothelial cells. J Clin Pathol 1994;47(3):263-265.

15. Fehm T, Beck EE, Valerius T, et al. CA 125 elevations in patients with malignant lymphomas. Tumour Biol 1998;19(4):283-289.

16. Revannasiddaiah S, Susheela SP, Thakur P, et al. Hodgkin's lymphoma involving extranodal sites: potential association with HIV infection and the implications for clinical management. Chin J Cancer 2013;32(2):102.

17. Yotsumoto M, Hagiwara S, Ajisawa A, et al. Clinical characteristics of human immunodeficiency virus-associated Hodgkin lymphoma patients in Japan. Int J Hematol 2012; 96(2):247-253.

18. Sissolak G, Sissolak D, Jacobs P. Human immunodeficiency and Hodgkin lymphoma. Transfus Apher Sci 2010;42(4): 131-139. 